\section{Künstliche Beatmung: besseres Überleben durch energiedichte enterale Ernährung?}

Chapman $\mathrm{M}$ et al. Energy-Dense versus Routine Enteral Nutrition in the Critically III. N Engl J Med 2018; 379: 1823 - 1834

Ärzte der ANZICS (Australian and New Zealand Intensive Care Society) entwarfen die binationale, multizentrische Studie TARGET, um die Hypothese zu prüfen, dass eine energiedichte enterale Ernährung bei beatmeten Patienten zu besseren 90-Tage-Überlebensraten führt als die üblicherweise empfohlene Menge.

An der TARGET (The Augmented versus Routine Approach to Giving Energy Trial)-Studie beteiligten sich 46 Intensivstationen (ICUs) in Australien und Neuseeland. Eingeschlossen wurden erwachsene Patienten, die eine Gerätebeatmung erhielten. Diese Patienten wurden im Verhältnis 1:1 randomisiert und erhielten entweder eine energiedichte Ernährung von $1,5 \mathrm{kcal}$ pro Milliliter oder die übliche Ernährung von $1,0 \mathrm{kcal}$ pro Milliliter. Die Nahrungszufuhr erfolgte so schnell als möglich nach Randomisierung. Angestrebt wurde eine Dosis von $1 \mathrm{ml}$ pro Kilogramm Körpergewicht pro Stunde. Um das Risiko der Überfütterung auszuschlieBen, wurde die Zufuhr auf $100 \mathrm{ml} /$ Stunde begrenzt. Empfohlen wurde eine Blutglukosekonzentration von $180 \mathrm{mg} / \mathrm{dl}$ oder weniger. Die Studienernährung wurde bis zu 28 Tagen, während sich der Patient auf der Intensivstation befand, zugeführt bzw. bis zum Tod oder der Entlassung von der Intensivstation. Primäres Studienergebnis war die Gesamtmortalität innerhalb von 90 Tagen.

In die modifizierte Intention-to-TreatAnalyse wurden 3957 Patientinnen und Patienten einbezogen, und zwar 1971 in der 1,5-kcal-Gruppe und 1986 in der 1,0-kcal-Gruppe. Zur Auswertung des primären Studienergebnisses standen Daten von 3914 Patienten (97,9\%) zur Verfügung. Die demografischen und klinischen Merkmale beider Gruppen waren ausgeglichen. Im Durchschnitt betrug die
Zeit zwischen Aufnahme auf die Intensivstation und Randomisierung 14,2 Stunden. Dauer der Ernährungszufuhr und Volumen der enteralen Ernährung, das während der Studie verabreicht wurde, waren in beiden Gruppen ähnlich. Patienten in der 1,5-kcal-Gruppe nahmen im Mittel 47,6\% mehr Kalorien zu sich als die Vergleichsgruppe. Sie erhielten demnach im Mittel $1863 \pm 478 \mathrm{kcal}$ pro Tag im Vergleich zu $1262 \pm 313 \mathrm{kcal}$ pro Tag in der 1,0-kcal-Gruppe (mittlerer Unterschied: $601 \mathrm{kcal}$ pro Tag; 95\%-Konfidenzintervall 576-626).

Am 90. Tag waren insgesamt 523 von 1948 Patienten $(26,8 \%)$ in der 1,5 -kcalGruppe und 505 von 1966 Patienten $(25,7 \%)$ in der 1,0 -kcal-Gruppe gestorben (relatives Risiko: 1,05; $95 \%$-KI: 0,941,16; $p=0,41)$. Die Ergebnisse waren in den 7 Untergruppen, stratifiziert nach Alter, Trauma (ja/nein), Sepsis (ja/nein), neurologischer Diagnose (ja/nein), Behandlung (medizinisch/operativ), Mortalitätsrisiko-Quintile sowie BMI, vergleichbar. Eine höhere Kalorienzufuhr hatte keinen Einfluss auf die Überlebenszeit, den Erhalt der Organunterstützung, auf die Zahl überlebter Tage außerhalb der Intensivstation und des Krankenhauses oder der Freiheit von einer Organunterstützung sowie der Häufigkeit infektiöser Komplikationen oder unerwünschter Ereignisse.

FAZIT

Die Studie zeigt, so das Autorenkomitee, dass bei Patienten, die eine Gerätebeatmung erhielten, die Überlebensrate nach 90 Tagen bei einer energiedichten enteralen Ernährungszufuhr nicht höher ausfiel als mit einer gewohnheitsmäßigen enteralen Ernährung.

Richard Kessing, Zeiskam 